University of Wollongong

Research Online

Faculty of Social Sciences - Papers (Archive) Faculty of Arts, Social Sciences \& Humanities

2019

Using principal components analysis to examine resting state EEG in relation to task performance

Diana Karamacoska

University of Wollongong, dk744@uowmail.edu.au

Robert J. Barry

University of Wollongong, rbarry@uow.edu.au

Genevieve Z. Steiner

University of Wollongong, gsteiner@uow.edu.au

Follow this and additional works at: https://ro.uow.edu.au/sspapers

Part of the Education Commons, and the Social and Behavioral Sciences Commons

Research Online is the open access institutional repository for the University of Wollongong. For further information contact the UOW Library: research-pubs@uow.edu.au 


\title{
Using principal components analysis to examine resting state EEG in relation to task performance
}

\author{
Abstract \\ Brain dynamics research has highlighted the significance of the ongoing EEG in ERP genesis and \\ cognitive functioning. Few studies, however, have assessed the contributions of the intrinsic resting state \\ EEG to these stimulus-response processes and behavioral outcomes. Principal components analysis \\ (PCA) has increasingly been used to obtain more objective, data-driven estimates of the EEG and ERPs. \\ PCA was used here to reassess resting state EEG and go/no-go task ERP data from a previous study \\ (Karamacoska et al., 2017) and the relationships between these measures. Twenty adults had EEG \\ recorded with eyes closed (EC) and eyes open (EO), and as they completed an auditory go/no-go task. \\ Separate EEG and ERP PCAs were conducted on each resting condition and stimulus type. For each state, \\ seven EEG components were identified within the delta-beta frequency range, and six ERP components \\ were obtained for go and no-go stimuli. Within the task, mean reaction time (RT) correlated positively with \\ go P2 amplitude and negatively with P3b positivity. Regressions revealed that greater EC delta-1 \\ amplitude predicted shorter mean RT, and larger alpha-3 amplitude predicted go P3b enhancement. \\ These findings demonstrate the immediate P2 and P3b involvement in decision making and response \\ control and the intrinsic EC delta-1 and alpha-3 amplitudes that underpin these processes.

\section{Disciplines} \\ Education | Social and Behavioral Sciences

\section{Publication Details} \\ Karamacoska, D., Barry, R. J. \& Steiner, G. Z. (2019). Using principal components analysis to examine \\ resting state EEG in relation to task performance. Psychophysiology, 56 (5), e13327-1-e13327-11.
}


Using Principal Components Analysis to Examine Resting State EEG in Relation to Task Performance

Diana Karamacoska ${ }^{1 *}$, Robert J. Barry ${ }^{1}$, Genevieve Z. Steiner ${ }^{1,2}$

${ }^{1}$ Brain \& Behaviour Research Institute and School of Psychology, University of Wollongong, Wollongong New South Wales 2522, Australia

${ }^{2}$ NICM Health Research Institute and Translational Health Research Institute (THRI), Western Sydney University, Penrith New South Wales 2751, Australia

*Corresponding author

Address: School of Psychology, University of Wollongong, Wollongong New South Wales 2522, Australia.

Email: dk744@uowmail.edu.au 


\begin{abstract}
Brain dynamics research has highlighted the significance of the ongoing EEG in ERP genesis and cognitive functioning. Few studies, however, have assessed the contributions of the intrinsic resting state EEG to these stimulus-response processes and behavioural outcomes. Principal components analysis (PCA) has increasingly been used to obtain more objective, data-driven estimates of the EEG and ERPs. PCA was used here to reassess resting state EEG and Go/NoGo task ERP data from a previous study (Karamacoska et al., 2017) and the relationships between these measures. Twenty adults had EEG recorded with eyes-closed (EC) and eyes-open (EO), and as they completed an auditory Go/NoGo task. Separate EEG and ERP PCAs were conducted on each resting condition and stimulus type. For each state, seven EEG components were identified within the delta-beta frequency range, and six ERP components were obtained for Go and NoGo stimuli. Within the task, mean reaction time (RT) correlated positively with Go P2 amplitude and negatively with P3b positivity. Regressions revealed greater EC delta-1 amplitude predicted shorter mean RT, and larger alpha-3 amplitude predicted Go P3b enhancement. These findings demonstrate the immediate $\mathrm{P} 2$ and $\mathrm{P} 3 \mathrm{~b}$ involvement in decision-making and response control, and the intrinsic EC delta-1 and alpha-3 amplitudes that underpin these processes.
\end{abstract}

Keywords: Brain Dynamics; Event-related potentials (ERPs); Electroencephalography (EEG); Principal Components Analysis (PCA); Cognition; Decision-making 
Using Principal Components Analysis to Examine Resting State EEG in Relation to Task Performance

\section{Introduction}

Two-choice response tasks, such as the Go/NoGo, stop-signal, and continuous performance test (CPT), are often employed to assess cognitive functioning via behavioural measures like reaction time (RT), response variability, and accuracy. In particular, reaction time variability (RTV) has become recognised as a marker of cognitive control efforts, as it has been reliably shown to inversely predict response accuracy rates (Bellgrove, Hester, \& Garavan, 2004; Karamacoska, Barry, \& Steiner, 2017; Karamacoska, Barry, Steiner, Coleman, \& Wilson, 2018; Simmonds et al., 2007). Our research focuses on understanding the neuronal activity underlying these behavioural processes, using both electroencephalogram (EEG) and event-related potential (ERP) measures.

ERPs mark the neuronal responses to stimuli, and components like the N1 and P3 have been linked to attentional and cognitive control processes (Herrmann \& Knight, 2001; Kok, 1997; Näätänen \& Picton, 1987; Polich, 2007; Verleger, Jaśkowski, \& Wascher, 2005). Specifically, faster and less variable responses to Go stimuli have been associated with larger parietal P3b amplitudes (Ramchurn, de Fockert, Mason, Darling, \& Bunce, 2014; Saville et al., 2011; Saville et al., 2012) and less slow wave (SW) positivity (Karamacoska, Barry, Steiner, 2018; Karamacoska, Barry, Steiner, Coleman et al., 2018). The accuracy in withholding responses to NoGo is generally linked to the frontal N2b component (Folstein \& Van Petten, 2008) and greater frontocentral P3a positivity (Fogarty, Barry, De Blasio, \& Steiner, 2018; Karamacoska, Barry, Steiner, 2018). These stimulus-response outcomes are also known to be influenced by the individual's EEG activity in the prestimulus (De Blasio \& Barry, 2013a, 2013b, 2018; De Blasio, Barry, \& Steiner, 2013; Mathewson, Gratton, Fabiani, Beck, \& Ro, 2009; Rahn \& Başar, 1993a, 1993b) and poststimulus periods (Fernández et al., 
2002; Harmony, Alba, Marroquin, \& Gonzalez-Frankenberger, 2009; Harmony et al., 1996; Karakaş, Erzengin, \& Başar, 2000; Sauseng, Griesmayr, Freunberger, \& Klimesch, 2010). These findings demonstrate the fundamental involvement of the EEG in ERP genesis (see also Başar, 1998, 1999; Guntekin \& Başar, 2016; Karakaş \& Barry, 2017).

Brain oscillation theory posits that ongoing EEG oscillations are necessary mechanisms for event-related brain dynamics where the amplitude or power of the frequency cycle of interest persists into the ERP waveform (Klimesch, Sauseng, Hanslmayr, Gruber, \& Freunberger, 2007). While these periods can be considered immediate determinants of responding, they also reflect an activated state of the brain required to meet task demands. As Raichle (2010) argues, these assessments make it difficult to determine the exact impact of the brain's intrinsic EEG activity on stimulus-response processes. An alternative approach is to examine the pre-task resting state EEG in relation to performance (Northoff, Duncan, \& Hayes, 2010).

Eyes-closed (EC) delta and theta band amplitudes have been shown to predict the N11 (Karamacoska, Barry, Steiner, Coleman et al., 2018) and P3b (Karamacoska et al., 2017) components of the ERP. Similar relationships were reported with eyes-open (EO) (Intriligator \& Polich, 1995; Polich, 1997), highlighting the involvement of the brain's low frequency activity in attention-related mechanisms. These studies demonstrate the relevance of examining resting state EEG to better understand the contributions of intrinsic activity to cognitive processes.

EEG changes, from EC to EO, have also generated scientific interest. Barry et al. (2007) noted that in the shift to EO, delta-alpha amplitudes decreased parietally and beta increased frontally, marking cortical adjustment to visual input. The posterior alpha decrease also correlated with an increase in arousal; and this measure was later examined by Tenke, Kayser, Abraham, Alvarenga, and Bruder (2015) for its effects during a novelty oddball task. 
Individuals with low EC to EO alpha reductions, that is, lower arousal increases, had greater prestimulus alpha levels and poststimulus alpha desynchronisation, revealing that the changes in baseline alpha rhythms persisted during stimulus-response processes. However, when the EC to EO changes in the traditional bands (delta, theta, alpha and beta) were modelled as predictors of ERP components in a Go/NoGo task, no effects were found (Karamacoska et al., 2017; Karamacoska, Barry, Steiner, Coleman et al., 2018).

Across the aforementioned studies, EEG band activity was assessed using predefined frequency ranges. While this approach is typical in EEG studies, it remains arbitrary in the choice of band limits leading to a lack of sensitivity and specificity. More sensitive estimations of the EEG have been proposed, such as calculating an individual's peak alpha frequency (Klimesch, Sauseng, \& Gerloff, 2003), but this too lacks objectivity. Instead, here we adopt a data-driven approach to decomposing the EEG - using frequency-PCA (f-PCA) to investigate the contributions of the resting state EEG to Go/NoGo task performance.

Tenke and Kayser (2005) utilised f-PCA in decomposing current source density (CSD) transformed EEG amplitude data from EC and EO resting states. Adopting the same parameters previously established for ERP temporal PCA (t-PCA; see Kayser \& Tenke, 2003), they submitted EEG data to unrestricted covariance-based PCA with Varimax rotation. Three posterior alpha components were identified, within the 9-11 Hz range, and showed the expected 'blockade'/reduction in alpha amplitude from EC to EO. This method was applied in subsequent studies examining the EEG in antidepressant treatment response (Tenke et al., 2011; Tenke, Kayser, Pechtel, et al., 2017) and spirituality (Tenke, Kayser, Svob, et al., 2017). In a recent application of f-PCA to both resting state and prestimulus task data, Barry and De Blasio (2018) found the Varimax rotation suboptimal, when compared with Promax rotated factors, due to the uninterpretable negative loadings obtained at some frequencies. Although Varimax is preferred for ERP decompositions (as it maintains 
orthogonality in components), the Promax rotation revealed EEG components to be highly correlated and argued that the underlying data were better estimated using this solution. The present study adopts Barry and De Blasio's approach to re-examine the resting state EEG data from our previous study (Karamacoska et al., 2017) and will assess the relationships between this activity and task-based response measures (i.e., ERP components and behavioural outcomes).

\subsection{Hypotheses}

We expected similar findings to be obtained here as in the original study (Karamacoska et al., 2017). Go/NoGo ERPs were decomposed using t-PCA, and f-PCA was implemented for the EC and EO resting EEG. Although the previous study utilised a single PCA across the two stimulus conditions, the current method uses an optimised approach, applying PCA separately on each of the conditions to minimise variance misallocation (Barry, De Blasio, Fogarty, \& Karamacoska, 2016). The following ERP components were anticipated to be extracted: The frontocentral N1-1 and temporal Processing Negativity (PN), a centrally dominant $\mathrm{P} 2$, a frontal N2c and parietal P3b specific to the Go stimulus, the frontocentral P3a and a second diffuse P3 to NoGo, and the bipolar slow wave (SW). The amplitudes of the P2, N2c, P3 and SW components were reassessed for their links to behavioural outcomes. It was anticipated that RTV would correlate positively with P2 amplitudes and mean RT would relate directly to Go N2c and correlate negatively with P3b amplitudes. Non-significant relationships between NoGo error rates and ERP components were expected here. For resting state intrinsic EEG, similar f-PCA outcomes as those identified by Barry and De Blasio (2018) were anticipated. These consisted of a frontocentral delta component, a second factor overlapping the delta/theta band ranges, three posterior alphas and two parietal-midline beta components. The change from EC to EO (termed reactivity) was also examined, with a reduction across delta-alpha amplitudes, and frontal 
increases in beta, expected (Barry et al., 2007; Barry \& De Blasio, 2018; Karamacoska et al., 2017). Based on the original study, EC delta was anticipated to positively predict Go P3b amplitude. Non-significant links between resting state EEG measures and behavioural outcomes were also reported and so we expected similar results here. EC to EO reactivity previously showed non-significant relationships with ERP component amplitudes and we expected to replicate these results here.

\section{Method}

The EEG/ERP data from Karamacoska et al. (2017) were re-processed in this study. A brief outline of the method is provided here, and further methodological details can be found in the original study. The study's protocol was approved by the local ethics committee.

\subsection{Participants}

Twenty right-handed university students ( 8 male) aged between 18 and 30 years, free of any head injury, neurological disorders, vision and hearing problems, provided written informed consent to participate. All self-reported abstinence from tobacco, caffeine, psychoactive substances, and alcohol for a minimum of 12 hours prior to participation.

\subsection{Task and Procedure}

Participants completed an electro-oculogram (EOG) calibration task, followed by 2 minute recordings of EC and EO resting state activity, and 2 blocks of the unwarned equiprobable Go/NoGo task. Each block consisted of 300 tones, half of which were randomly presented at $1000 \mathrm{~Hz}$ and the other at $1500 \mathrm{~Hz}$, each $80 \mathrm{~ms}$ (including $15 \mathrm{~ms}$ rise/fall) in duration at $60 \mathrm{~dB}$ SPL. The stimulus onset asynchrony (SOA) varied randomly between 1.0 and $1.5 \mathrm{~s}$. Participants were instructed to press a button to the Go tone of each block with their right index finger; Go tone frequencies were counterbalanced between blocks 
and participants. Throughout the EO and Go/NoGo task recordings, participants fixated on a white cross in the centre of the display.

\subsection{Electrophysiological Recording and Pre-Processing}

Continuous EEG from 30 electrodes (Fp1, Fp2, F7, F3, Fz, F4, F8, FT7, FC3, FCz, FC4, FT8, T7, C3, Cz, C4, T8, TP7, CP3, CPz, CP4, TP8, P7, P3, Pz, P4, P8, O1, Oz, O2) and A2 were recorded on a Neuroscan Synamps 2 system. The cap was grounded by an electrode positioned in the middle of $\mathrm{Fz}$ and $\mathrm{Fp} 1 / \mathrm{Fp} 2$, with $\mathrm{A} 1$ as the active reference. Vertical and horizontal EOGs were also recorded. All electrodes were tin and impedance levels were $<5 \mathrm{k} \Omega$. Data were sampled DC to $70 \mathrm{~Hz}$, and digitized at a rate of $1 \mathrm{kHz}$. Data were then processed offline to correct for eye movements, using the revised aligned-artefact average (RAAA) EOG Correction Program (Croft \& Barry, 2000), and the EOG-corrected data were re-referenced to the average of digitally-linked ears.

\subsection{Task Data and ERP Processing}

The task-related EEG data had a low pass $30 \mathrm{~Hz}$ filter (zero-phase shift, 24 $\mathrm{dB} /$ Octave) applied and epochs were derived -100 to $600 \mathrm{~ms}$ around stimuli, baselined to the prestimulus period. Epochs were rejected if amplitudes exceeded $\pm 75 \mu \mathrm{V}$ at any site. Trials with NoGo commission errors, Go omission errors, or extreme RTs ( $\leq 150 \mathrm{~ms}$ or $\geq 800 \mathrm{~ms}$ ) were excluded, as were the trials that immediately followed these rejected epochs. Error rates were recorded for analysis. For the remaining Go epochs, mean RTs were calculated and only those within $1 \mathrm{SD}$ of this mean were accepted. RTV, measured as the within-subject SD of accepted RTs across these trials, was also recorded.

2.4.1 Temporal principal components analysis (t-PCA). Go and NoGo ERPs were formed using the remaining accepted epochs and submitted to t-PCA, using Dien's PCA toolkit (v. 2.23; Dien, 2010). The data from the 30 scalp sites were half-sampled to 350 timepoints/variables. Separate temporal PCAs were conducted for Go and NoGo ERPs 
(following Barry et al., 2016) with 600 cases in each. The covariance matrix and Kaiser normalisation were used, and all 350 factors were orthogonally rotated with Kayser and Tenke's (2003) version of Varimax4M. Following rotation, t-PCA factors that contributed $\geq$ $2 \%$ of the variance were selected for identification as ERP components according to their latency, topography, polarity, and sequence within the expected processing schema (Borchard, Barry, \& De Blasio, 2015; Karamacoska et al., 2017). The selected components were extracted and analysed at their region of maximal activity. The maximal site was identified in the PCA toolkit and the region of interest (pooled across 3 sites) was confirmed with the grand mean topographic illustrations of the voltage headmaps and their contour lines.

\subsection{Resting State EEG}

One second epochs were extracted from the two minutes of each resting EEG condition. Epochs were zeroed across this period and checked for activity at all sites exceeding $\pm 75 \mu \mathrm{V}$. MATLAB ${ }^{\circledR}$ was used to apply a $10 \%$ Hanning window to each EEG epoch. Discrete Fourier transformations were performed on the 1000 data points, obtaining 1 Hz resolution, with a correction applied for having used the window. Participants' mean EEG spectral amplitudes from each resting state (EC and EO), DC to $29 \mathrm{~Hz}$, were then submitted to f-PCA.

2.5.1 Frequency principal components analysis (f-PCA). Following Barry and De Blasio's (2018) f-PCA approach, all data (20 participants $\times 30$ sites $\times 2$ conditions $)$ were submitted to a PCA in Dien's toolkit using the covariance matrix and Kaiser normalisation with unrestricted Promax rotation on the 30 frequency points. This initial f-PCA was used to identify the major frequency components in the EEG data. To gain better estimates of component variance for each resting state, separate f-PCAs were then conducted (Barry et al., 2016) using the same parameters as the initial f-PCA. Each separate f-PCA contained 600 
cases (20 participants $\times 30$ sites $)$ and 30 components. All factors were extracted and rotated, and those contributing $\geq 1.5 \%$ of variance were assessed and labelled with reference to their peak frequency and topography.

\subsection{Statistical Analyses}

To compare reactivity between EC and EO resting states, EC components were assessed for topographic and spectral consistency with EO components. Two-way Pearson correlations were conducted using the topographic amplitudes from the 30 scalp sites, with $r(28)$ degrees of freedom. Unscaled f-PCA factor loadings were then assessed using Tucker's (1951) congruence coefficient $\left(r_{\mathrm{c}}\right)$ using an accepted rule of thumb, where $r_{\mathrm{c}}>.95$ indicates component equality, $r_{\mathrm{c}}>.80$ signifies fair similarity and $r_{\mathrm{c}}<.80$ reflects dissimilarity (Lorenzo-Seva and ten Berge, 2006). Factors showing dissimilarity were excluded from further analysis.

EEG component amplitudes for the EC state were then assessed to define the maximal regions of component activity. Topographies were assessed using separate within-subjects repeated measures MANOVAs involving 9 sites across the frontal (F: F3, Fz, F4), central (C: $\mathrm{C} 3, \mathrm{Cz}, \mathrm{C} 4)$, and parietal (P: P3, Pz, P4) regions. Planned orthogonal contrasts were conducted where the frontal $(\mathrm{F})$ and parietal $(\mathrm{P})$ regions were compared, and the frontoparietal (F/P) mean was contrasted against the central mean (C); the left (L: F3, C3, P3) and right (R: F4, C4, P4) hemispheres were contrasted, as was the midline (M: Fz, Cz, Pz) against the mean of the hemispheres (L/R). Bonferroni-type $\alpha$ adjustments were not required as these planned contrasts do not exceed the degrees of freedom for effect (Tabachnick \& Fidell, 2013). All $F$ tests reported had $(1,19)$ degrees of freedom. Violations of sphericity assumptions do not affect MANOVAs with single degree of freedom contrasts and so Greenhouse-Geisser-type corrections were not necessary (O’Brien \& Kaiser, 1985). EC to EO reactivity was also assessed for the EEG components that were congruent between the 
two datasets. The same $3 \times 3$ MANOVAs were conducted with the addition of the within subjects factor of state (EC, EO). The maximal regions of band activity were identified based on these analyses and analysed as the average of the adjacent electrodes from the broader 30 site array. The outcomes of these MANOVAs are presented in Supplementary Materials, with a brief outline of the selected ROI provided in the results.

Performance patterns involving relations between ERP components and behavioural outcomes were re-examined with the amplitude data obtained from the separate t-PCAs. As similar relationships were expected here (cf. Karamacoska et al., 2017), one-tailed Pearson correlations $(r)$ were conducted with $r(18)$ degrees of freedom.

Separate stepwise multiple regressions were then conducted to determine the impacts of the EC intrinsic EEG on Go/NoGo performance measures. The regional maxima of each EEG component measure were entered as predictors of unique variance in the dependent variables of Go/NoGo error rates, mean RTs, and RTV, and the P2, P3 and SW ERP components. A second set of regressions were then run for the same dependent variables with EC to EO reactivity measures entered as predictors. As two sets of regressions were run for each dependent variable, significance levels were set at the Bonferroni-corrected alpha level of 0.025 .

\section{Results}

\subsection{Go/NoGo Task Outcomes}

Go/NoGo error rates were low in this task (Go omissions ranged from $0-7.3 \%, M=$ $1.5 \pm 1.9 \%$; NoGo commissions ranged from $0-9.0 \%, M=2.8 \pm 2.4 \%$ ) and extreme RTs were minimal ( $\leq 5 \%$ of trials per participant). Go Mean RT ranged from $291.1-437.8 \mathrm{~ms}$ ( $M$ $=376.1 \pm 38.9 \mathrm{~ms})$ and RTV ranged from $24.8-61.4 \mathrm{~ms}(M=45.9 \pm 9.9 \mathrm{~ms})$.

\subsection{Go/NoGo ERPs and t-PCA Outcomes}


For Go ERPs, an average of $194(S D=18)$ epochs were accepted and for NoGo ERPs, an average of $263(S D=24)$ were accepted per subject. Grand mean ERPs, at the midline sites, are presented in the top panels of Figures 1 and 2. Of the 350 factors rotated in the t-PCAs, the first 6 each carried $>2.1 \%$ of variance and were identified as major ERP components, with over $87 \%$ of the variance explained in each dataset. The PCAreconstituted ERPs (dashed lines in Figures 1 and 2 top panel) show a good fit with the original data.

The following components were extracted from the Go t-PCA: The N1-1 (dominant across Fz, FCz and Cz), PN (maximal across FT8, T8 and TP8), P2 (maximal at $\mathrm{Cz}$ and averaged across $\mathrm{FCz}, \mathrm{Cz}$ and $\mathrm{CPz}$ ), a complex at $312 \mathrm{~ms}$ comprising the overlapping frontal Go N2c and parietal P3; for consistency with our previous study only the negativity was assessed (dominant across F3, Fz and F4), the posterior-left dominant Go P3b was also identified (maximal over CP3, P3 and Pz), as was the bipolar SW but only the central positivity was analysed (largest across $\mathrm{CP} 3, \mathrm{CPz}$ and $\mathrm{CP} 4)$.

\section{INSERT FIGURE 1 ABOUT HERE.}

In the NoGo t-PCA, the following components were identified: The N1-1 (dominant across F3, Fz, and F4), PN (maximal across F4, F8 and FC4), P2 (pooled across FCz, Cz and $\mathrm{CPz}$ ), a NoGo $\mathrm{P} 3 \mathrm{a}$ (largest at $\mathrm{FCz}$ with positivity pooled from $\mathrm{FCz}, \mathrm{Cz}$ and $\mathrm{CPz}$ ), a second right-hemispheric P3 (maximal at CP4; averaged across C4, CP4 and P4), and the bipolar SW with a prominent centroparietal positivity (largest over $\mathrm{CP} 3, \mathrm{CPz}$ and $\mathrm{CP} 4$ ).

\section{INSERT FIGURE 2 ABOUT HERE.}

\subsection{Resting State EEG and f-PCA Outcomes}

Grand mean spectral EEG amplitudes, at the midline sites, from 0 to $29 \mathrm{~Hz}$ for the EC and EO resting states can be viewed in Figure 3. Prominent peaks in the delta and alpha bands can be seen with a notable decrease in alpha amplitude from EC to EO. The first 7 
factors from each f-PCA carried more than $1.5 \%$ of variance and had similar peak frequencies and topographic distributions (see Supplementary Materials Figure S1 for factor information from each f-PCA). Condition-based variance misallocation was confirmed in the initial f-PCA (see Supplementary Materials S1.1), and so data from the separate f-PCAs were utilised in subsequent assessments. One prominent delta component was extracted at $1 \mathrm{~Hz}$ (delta-1), followed by an overlapping delta/theta component that peaked predominantly at 1 $\mathrm{Hz}$ in the EC state and $2 \mathrm{~Hz}$ with EO; with a second peak in the theta range (4 Hz) in both conditions. Three alpha components were extracted, and each shifted by $1 \mathrm{~Hz}$ in the change from EC to EO. Two beta components were also identified: beta-1 shifted from $15 \mathrm{~Hz}$ with EC to $18 \mathrm{~Hz}$ with EO, and beta-2 remained stable at $27 \mathrm{~Hz}$.

\section{INSERT FIGURE 3 ABOUT HERE.}

As shown in the bottom panel of Figure 3, all EC and EO components from the separate f-PCAs had topographic consistency with scalp amplitudes correlating between $r \geq$ .80 and $\leq .98$, all $p<0.001$. When factor loadings were compared, the following were found to be fairly congruent $\left(r_{\mathrm{c}} \geq .87\right)$ : delta- 1 , delta/theta, alpha-1 and beta- 2 . These EEG components were retained for further analysis of EC to EO reactivity.

3.3.1 EEG component topographies. Table S1 in Supplementary Materials presents the topographic MANOVA outcomes for the EEG components for the EC state and the change from EC to EO. The ROI for each EC EEG component was identified as follows: delta-1 and delta/theta activity was pooled across the dominant $\mathrm{FCz}, \mathrm{Cz}$, and $\mathrm{CPz}$ sites. All three alphas, and beta-1, were posterior dominant, and so the ROI was defined as the average over P3, Pz, and P4. Beta-2 was maximal over the midline region and pooled from $\mathrm{FCz}, \mathrm{Cz}$ and $\mathrm{CPz}$.

EC to EO reactivity was marked by an overall increase in delta-1 amplitude; for consistency with EC, the midline region was selected $(\mathrm{FCz}, \mathrm{Cz}$, and $\mathrm{CPz})$. Delta/theta 
decreased largely in the midline and so this became the ROI. Alpha-1 showed a strong parietal decrease, and so P3, Pz, and P4 were selected for the ROI. Beta-2 amplitude increased from EC to EO, predominantly in the frontal hemispheres (F3 and F4). With the ROIs identified, the amplitude difference between EC and EO at these sites was calculated and then averaged to provide a measure of that reactivity.

\subsection{ERP Correlates of Behaviour}

The stimulus-specific P2 to SW component amplitudes, at their maximal regions, were assessed for their relations with the corresponding behavioural outcomes. NoGo component amplitudes did not correlate significantly with commission error rates (all $r \leq-$ $0.28, p \geq 0.240)$. Table 1 displays the correlations between the Go-related ERP components and measures of omissions, mean RT, and RTV.

\section{INSERT TABLE 1 ABOUT HERE.}

\subsection{EC EEG and Go/NoGo Performance}

Prior to the multiple regressions being conducted, collinearity between the EC EEG variables was checked. The highest correlations were between alpha-2 and alpha-3 amplitudes $(r=0.58, p=0.008)$ and between alpha- 2 and beta- 1 amplitudes $(r=0.62, p=$ $0.003)$; all other variables were moderately correlated $(|r| \leq 0.50, p \geq 0.023)$. As alpha-2 was common to these relationships, it was excluded from the regression models. Separate stepwise regressions modelled the regional EC activity of the six EEG components (delta-1, delta/theta, alpha-1, alpha-3, beta-1, and beta-2) as predictors of Go/NoGo behavioural performance and ERP component amplitudes (Go P2, P3b, SW; NoGo P2, P3a, P3 and SW).

No significant models were obtained for Go error rates and RTV, or for Go N1-1, P2, and SW amplitudes; nor for NoGo error rates, P3a or SW positivity. Table 2 shows the significant EC EEG predictors of Go mean RT and P3b. Mean RT was negatively predicted by EC delta- 1 amplitude, accounting for $20.3 \%$ of the variance $(p=0.023)$ EC alpha-3 
component amplitude positively predicted P3b amplitude, explaining $29.1 \%$ of the variance $(p=0.007)$. This relationship differs from our expected delta-P3b finding but when alpha-3 was removed as a predictor, a positive relationship between the delta/theta component and P3b amplitude was found, explaining $17.6 \%$ of the variance $(p=0.032)$. However, it should be noted that this relationship did not reach statistical significance according to the Bonferroni-adjusted alpha level.

INSERT TABLE 2 ABOUT HERE.

\subsubsection{EC to EO Reactivity Relations to Task Outcomes}

The next set of stepwise regressions had EC to EO reactivity in the delta-1, delta/theta, alpha- 1 and beta- 2 components entered as predictors of the same dependent measures as the previous tests. EC to EO reactivity in the delta-1, delta/theta, alpha-1, and beta- 2 components were found to be moderately correlated $(|r| \leq 0.49, p \geq 0.027)$, and so all predictors were used in each model. There were no significant models found for the Go/NoGo behavioural outcomes and ERP component amplitudes.

\section{Discussion}

The current study revisited data from Karamacoska et al. (2017) and utilised PCA to better estimate ERP/EEG activity. In the original study, a single temporal PCA was used to decompose ERP data from both stimulus types. This approach has been argued to misallocate the variance between conditions (see Barry et al., 2016). Although similar Go and NoGo ERP components were identified, as in the original study, the reconstituted data from the separate PCAs reflected a better fit with the input data. For resting state EEG, similar components were extracted as in Barry and De Blasio (2018). One prominent delta component was identified, followed by a delta/theta component, three alphas and two betas. However, between-condition variance misallocation was apparent in the initial f-PCA conducted with both EC and EO resting states. As the condition factor was removed with the 
separate PCAs, the only source of variance came from within the condition. As such, the components extracted from each PCA better represented the data. This was further evidenced with the low congruence between the alpha-3, beta- 1 , and especially alpha- 2 components extracted from the separate resting states. This indicates an energetic shift in EEG activity in this frequency range resulting in components that are not alike and would otherwise be treated as singular in the initial f-PCA. These outcomes represent a more objective and datadriven estimate of the EEG than using the traditionally-divided four bands (as in Karamacoska et al., 2017), or further subdivided high/low alpha-beta range activity (Karamacoska, Barry, Steiner, Coleman et al., 2018; see also Intriligator and Polich, 1995; Polich, 1997). Thus, the simplified PCA structures of the brain's ERP/EEG activity allowed for better insights into the neuronal activity underpinning stimulus-response processes. The following discussion will address findings concerning the ERP component correlations with behavioural outcomes and the resting state EEG relations to these task-based measures.

When ERP component amplitudes were correlated with behavioural outcomes, several expected relationships were identified. RTV correlated positively with central Go P2 amplitude, mean RT correlated negatively with Go P3b positivity, and NoGo ERP components did not correlate with NoGo error rates. These findings remain consistent with Karamacoska et al. (2017), reaffirming the links between these Go ERP components and decision-making and response execution processes, and the lack of cognitive control required for NoGo stimuli in this paradigm (see also Borchard et al., 2015). Unexpectedly, mean RT also correlated positively with Go P2 enhancements, and a non-significant relationship between mean RT and Go N2c negativity was found. The different PCA methods between the studies can account for these results. While the Go P2 appeared to have been estimated better here, the $\mathrm{N} 2 \mathrm{c}$ component was extracted at a later latency (by $\sim 30 \mathrm{~ms}$ cf. original study) and overlapped with an ongoing P3b. Nevertheless, the direction of the relationship between 
mean RT and N2c negativity matched that of Karamacoska et al. (2017), rendering it a comparable finding.

To determine the impact of intrinsic neuronal activity on performance, resting state EEG component amplitudes were assessed for their effects on task-based measures (i.e., ERPs and behavioural outcomes). Two significant regression models were found with greater EC delta-1 amplitude predictive of shorter mean RTs, and larger EC alpha-3 amplitude predicting Go $\mathrm{P} 3 \mathrm{~b}$ enhancement. These findings differ from our previous study, as only a delta-P3b relationship was identified. The current delta-mean RT finding is not entirely unexpected, as mean RT has been shown to inversely relate to Go P3b positivity, noting this component's association with decision-making and response processes (Hogan et al., 2006; Ramchurn et al., 2014; Donchin \& Lindsley, 1966). Delta's role in attentionrelated mechanisms may therefore also extend to affect response control efforts. A similar notion was suggested by Karamacoska, Barry and Steiner (2018), as larger prestimulus delta amplitudes predicted longer mean RTs. The directional difference in these relationships suggests that delta functioning varies between resting and task-based states. Across our studies, delta amplitude was reported to increase from the resting state to the task, and this change consistently predicted poorer performance outcomes (see Karamacoska, Barry, Steiner, 2018; Karamacoska, Barry, Steiner, Coleman et al., 2018). This evidence supports the presence of functionally distinct delta activity, and has significant implications when interpreting findings obtained from the different states. While greater resting state delta may be useful in predicting better performance, larger prestimulus amplitudes may indicate lapses in attention and decision-making that detrimentally affect response outcomes.

The alpha-3 and P3b effect reported here is also novel. Although this finding is comparable to prior studies indicating a direct relationship between P3b amplitude and broadrange (8-13 Hz) alpha (De Blasio \& Barry, 2013b; De Blasio et al., 2013), and also 
subdivided alpha power (Intriligator \& Polich, 1995; Polich, 1997), it is the first report involving a resting state f-PCA component. Barry and De Blasio (2018) did not examine resting state EEG components in relation to ERPs, focusing only on the prestimulus EEG components. In their study, distinct P3b effects were found: Prestimulus alpha-1 and alpha-3 were inversely related to $\mathrm{P} 3 \mathrm{~b}$ amplitude, while alpha-2 directly predicted $\mathrm{P} 3 \mathrm{~b}$ enhancement. Given the known differences between resting state and task-based EEG, these results cannot be directly compared with the present one. Consideration must also be given as to the function of the different alphas. Prior work dissociating alpha into lower $(8-10 \mathrm{~Hz})$ and upper (11-13 Hz) bands links lower alpha to arousal (Loo et al., 2009) and upper alpha with memory-related processes (Bazanova \& Vernon, 2014; Klimesch, Schack, \& Sauseng, 2005). With the novel identification of three alphas, and their varying impacts on the P3, additional research is needed into their functional significance.

In line with expectations, EC to EO reactivity was not related to task outcomes. Although Tenke et al. (2015) were able to demonstrate a relationship between this measure of broad alpha change and task-based activity, it does not correspond to any ERP or behavioural effects. We have consistently found non-significant relationships between these measures suggesting that this change does not have a meaningful impact on Go/NoGo stimulusresponse processes.

While this study replicated the ERP-behavioural correlations obtained in the original investigation, the implementation of f-PCA to decompose EEG data resulted in novel relationships being identified. Together, these findings highlight the roles of the P2 and P3b components in response control efforts, and the intrinsic EC delta-1 and alpha-3 amplitudes that affect these behavioural processes. As this is the first EEG-ERP study to conduct f-PCA separately on the resting state conditions using Promax rotation, comparisons with prior work are limited and so replication is required. Variance misallocation was clearly evident when 
all conditions were included in the PCA, and while the single condition PCA approach has been established for ERPs, further validation is needed for EEG. Future investigations into EEG component functionalities are also warranted, particularly in dissociating their significance between resting and task-based states. The application of PCA in the ERP and EEG domains continues efforts to understand the dynamics between these measures and their relevance to cognitive functioning and behavioural output. 


\section{References}

Barry, R. J., Clarke, A. R., Johnstone, S. J., Magee, C. A., \& Rushby, J. A. (2007). EEG differences between eyes-closed and eyes-open resting conditions. Clinical Neurophysiology, 118(12), 2765-2773. https://doi.org/10.1016/j.clinph.2007.07.028

Barry, R. J., \& De Blasio, F. M. (2018). EEG frequency-PCA in EEG-ERP dynamics. Psychophysiology, 55, e13042. https://doi.org/10.1111/psyp.13042

Barry, R. J., De Blasio, F. M., Fogarty, J. S., \& Karamacoska, D. (2016). ERP Go/NoGo condition effects are better detected with separate PCAs. International Journal of Psychophysiology, 106, 50-64. https://doi.org/10.1016/j.ijpsycho.2016.06.003

Başar, E. (1998). Brain function and oscillations: Brain oscillations principles and approaches (Vol. 1). Berlin: Springer.

Başar, E. (1999). Brain function and oscillations: II. Integrative brain function, neurophysiology and cognitive processes. Berlin: Springer.

Bazanova, O. M., \& Vernon, D. (2014). Interpreting EEG alpha activity. Neuroscience and Biobehavioral Reviews, 44, 94-110. https://doi.org/10.1016/j.neubiorev.2013.05.007

Bellgrove, M. A., Hester, R., \& Garavan, H. (2004). The functional neuroanatomical correlates of response variability: evidence from a response inhibition task. Neuropsychologia, 42(14), 1910-1916. https://doi.org/10.1016/j.neuropsychologia.2004.05.007

Borchard, J. P., Barry, R. J., \& De Blasio, F. M. (2015). Sequential processing in an auditory equiprobable Go/NoGo task with variable interstimulus interval. International Journal of Psychophysiology, 97(2), 145-152. https://doi.org/10.1016/j.ijpsycho.2015.05.010

Croft, R. J., \& Barry, R. J. (2000). Removal of ocular artefact from the EEG: A review. Clinical Neurophysiology, 30, 5-19. https://doi.org/10.1016/S0987-7053(00)00055-1 
De Blasio, F. M., \& Barry, R. J. (2013a). Prestimulus alpha and beta determinants of ERP responses in the Go/NoGo task. International Journal of Psychophysiology, 89(1), 917. https://doi.org/10.1016/j.ijpsycho.2013.04.018

De Blasio, F. M., \& Barry, R. J. (2013b). Prestimulus delta and theta determinants of ERP responses in the Go/NoGo task. International Journal of Psychophysiology, 87(3), 279-288. https://doi.org/10.1016/j.ijpsycho.2012.09.016

De Blasio, F. M., \& Barry, R. J. (2018). Prestimulus delta and theta contributions to equiprobable Go/NoGo processing in healthy ageing. International Journal of Psychophysiology, 130, 40-52. https://doi.org/10.1016/j.ijpsycho.2018.05.005

De Blasio, F. M., Barry, R. J., \& Steiner, G. Z. (2013). Prestimulus EEG amplitude determinants of ERP responses in a habituation paradigm. International Journal of Psychophysiology, 89(3), 444-450. https://doi.org/10.1016/j.ijpsycho.2013.05.015

Dien, J. (2010). The ERP PCA toolkit: An open source program for advanced statistical analysis of the event-related potential data. Journal of Neuroscience Methods, 187, 138-145. https://doi.org/10.1016/j.jneumeth.2009.12.009

Donchin, E., \& Lindsley, D. B. (1966). Averaged evoked potentials and reaction times to visual stimuli. Electroencephalography and Clinical Neurophysiology, 20, 217-223. https://doi.org/10.1016/0013-4694(66)90086-1

Fasmer, O.B., Mjeldheim, K., Forland, W., Hansen, A.L., Syrstad, V.E., Oedegaard, K.J., Berle, J.O., 2016. Linear and non-linear analyses of Conner's Continuous Performance Test-II discriminate adult patients with attention deficit hyperactivity disorder from patients with mood and anxiety disorders. BMC Psychiatry, 16, 284294. https://doi.org/10.1186\%2Fs12888-016-0993-4

Fernández, T., Harmony, T., Silva-Pereyra, J., Fernández-Bouzas, A., Gersenowies, J., Galán, L., . . . Valdés, S. I. (2000). Specific EEG frequencies at specific brain areas and 
performance. Cognitive Neuroscience and Neuropsychology, 11, 2663-2668. https://doi.org/10.1002/cam4.1016

Fogarty, J. S., Barry, R. J., De Blasio, F. M., \& Steiner, G. Z. (2018). ERP components and behavior in the auditory equiprobable go/no-go task: Inhibition in young adults. Psychophysiology. https://doi.org/10.1111/psyp.13065

Folstein, J. R., \& Van Petten, C. (2008). Influence of cognitive control and mismatch on the N2 component of the ERP: A review. Psychophysiology, 45, 152-170. https://doi.org/10.1111/j.1469-8986.2007.00602.x

Guntekin, B., \& Başar, E. (2016). Review of evoked and event-related delta responses in the human brain. International Journal of Psychophysiology, 103, 43-52. https://doi.org/10.1016/j.ijpsycho.2015.02.001

Harmony, T., Alba, A., Marroquin, J. L., \& Gonzalez-Frankenberger, B. (2009). Timefrequency-topographic analysis of induced power and synchrony of EEG signals during a Go/No-Go task. International Journal of Psychophysiology, 71, 9-16. https://doi.org/10.1016/j.ijpsycho.2008.07.020

Harmony, T., Fernández, T., Silva, J., Bernal, J., Diaz-Comas, L., Reyes, A., . . Rodriguez, M. (1996). EEG delta activity: an indicator of attention to internal processing during performance on mental tasks. International Journal of Psychophysiology, 24, 161171. https://doi.org/10.1016/S0167-8760(96)00053-0

Herrmann, C. S., \& Knight, R. T. (2001). Mechanisms of human attention: Event-related potentials and oscillations. Neuroscience and Biobehavioral Reviews, 25, 465-476. https://doi.org/10.1016/S0149-7634(01)00027-6

Hogan, M. J., Carolan, L., Roche, R. A., Dockree, P. M., Kaiser, J., Bunting, B. P., . . . Lawlor, B. A. (2006). Electrophysiological and information processing variability predicts memory decrements associated with normal age-related cognitive decline and 
Alzheimer's disease (AD). Brain Research, 1119, 215-226.

https://doi.org/10.1016/j.brainres.2006.08.075

Intriligator, J., \& Polich, J. (1995). On the relationship between EEG and ERP variability. International Journal of Psychophysiology, 20, 59-74. https://doi.org/10.1016/01678760(95)00028-Q

Karakaş, S., \& Barry, R. J. (2017). A brief historical perspective on the advent of brain oscillations in the biological and psychological disciplines. Neuroscience and Biobehavioral Reviews, 75, 335-347. https://doi.org/10.1016/j.neubiorev.2016.12.009

Karakaş, S., Erzengin, O. U., \& Başar, E. (2000). A new strategy involving multiple cognitive paradigms demonstrates that ERP components are determined by the superposition of oscillatory responses. Clinical Neurophysiology, 111, 1719-1732. https://doi.org/10.1016/S1388-2457(00)00418-1

Karamacoska, D., Barry, R. J., \& Steiner, G. Z. (2017). Resting state intrinsic EEG impacts on go stimulus-response processes. Psychophysiology, 54, 894-903. https://doi.org/10.1111/psyp.12851

Karamacoska, D., Barry, R. J., Steiner, G. Z., Coleman, E. P., \& Wilson, E. J. (2018). Intrinsic EEG and task-related changes in EEG affect Go/NoGo task performance. International Journal of Psychophysiology, 125, 17-28. https://doi.org/https://doi.org/10.1016/j.ijpsycho.2018.01.015

Karamacoska, D., Barry, R. J., \& Steiner, G. Z. (2018). Electrophysiological underpinnings of response variability in the Go/NoGo task. International Journal of Psychophysiology, 134, 159-167. https://doi.org/10.1016/j.ijpsycho.2018.09.008

Kayser, J., \& Tenke, C. E. (2003). Optimizing PCA methodology for ERP component identification and measurement: Theoretical rationale and empirical evaluation. 
Clinical Neurophysiology, 114(12), 2307-2325. https://doi.org/10.1016/s13882457(03)00241-4

Klimesch, W., Sauseng, P., \& Gerloff, C. (2003). Enhancing cognitive performance with repetitive transcranial magnetic stimulation at human individual alpha frequency. European Journal of Neuroscience, 17(5), 1129-1133. https://doi.org/10.1046/j.14609568.2003.02517.x

Klimesch, W., Sauseng, P., Hanslmayr, S., Gruber, W., \& Freunberger, R. (2007). Eventrelated phase reorganization may explain evoked neural dynamics. Neuroscience and Biobehavioral Reviews, 31(7), 1003-1016. https://doi.org/10.1016/j.neubiorev.2007.03.005

Klimesch, W., Schack, B., \& Sauseng, P. (2005). The functional significance of theta and upper alpha oscillations. Experimental Psychology, 52(2), 99-108. https://doi.org/10.1027/1618-3169.52.2.99

Kok, A. (1997). Event-related potential (ERP) reflections of mental resources: A review and synthesis. Biological Psychology, 45, 19-56. https://doi.org/10.1016/S03010511(96)05221-0

Loo, S. K., Hale, T. S., Macion, J., Hanada, G., McGough, J. J., McCracken, J. T., \& Smalley, S. L. (2009). Cortical activity patterns in ADHD during arousal, activation and sustained attention. Neuropsychologia, 47, 2114-2119. https://doi.org/10.1016/j.neuropsychologia.2009.04.013

Mathewson, K. E., Gratton, G., Fabiani, M., Beck, D. M., \& Ro, T. (2009). To see or not to see: Prestimulus alpha phase predicts visual awareness. Journal of Neuroscience, 29(9), 2725-2732. https://doi.org/10.1523/JNEUROSCI.3963-08.2009 
Näätänen, R., \& Picton, T. (1987). The N1 wave of the human electric and magnetic respond to sound: A review and analysis of component structure. Psychophysiology, 24, 375425. https://doi.org/10.1111/j.1469-8986.1987.tb00311.x

Northoff, G., Duncan, N. W., \& Hayes, D. J. (2010). The brain and its resting state activityexperimental and methodological implications. Progress in Neurobiology, 92(4), $593-$ 600. https://doi.org/10.1016/j.pneurobio.2010.09.002

O'Brien, R. G., \& Kaiser, M. K. (1985). MANOVA method for analysing repeated measures designs: An extensive primer. Psychological Bulletin, 97, 316-333. https://doi.org/10.1037/0033-2909.97.2.316

Polich, J. (1997). EEG and ERP assessment of normal aging. Electroencephalography and Clinical Neurophysiology, 104, 244-256. https://doi.org/10.1016/S01685597(97)96139-6

Polich, J. (2007). Updating P300: An integrative theory of P3a and P3b. Clinical Neurophysiology, 118(10), 2128-2148. https://doi.org/10.1016/j.clinph.2007.04.019

Rahn, E., \& Başar, E. (1993a). Enhancement of visual evoked potentials by stimulation during low prestimulus EEG stages. The International Journal Of Neuroscience, 72 , 123-136. https://doi.org/10.3109/00207459308991629

Rahn, E., \& Başar, E. (1993b). Prestimulus EEG activity strongly influences the auditory evoked vertex response: A new method for selective averaging. International Journal of Neuroscience, 69(1-4), 207-220. https://doi.org/10.3109/00207459309003331

Raichle, M. E. (2010). Two views of brain function. Trends in Cognitive Sciences, 14(4), 180-190. https://doi.org/10.1016/j.tics.2010.01.008

Ramchurn, A., de Fockert, J. W., Mason, L., Darling, S., \& Bunce, D. (2014). Intraindividual reaction time variability affects $\mathrm{P} 300$ amplitude rather than latency. Frontiers in Human Neuroscience, 8, 557. https://doi.org/10.3389/fnhum.2014.00557 
Sauseng, P., Griesmayr, B., Freunberger, R., \& Klimesch, W. (2010). Control mechanisms in working memory: A possible function of EEG theta oscillations. Neuroscience and Biobehavioral Reviews, 34(7), 1015-1022. https://doi.org/10.1016/j.neubiorev.2009.12.006

Saville, C. W., Dean, R. O., Daley, D., Intriligator, J., Boehm, S., Feige, B., \& Klein, C. (2011). Electrocortical correlates of intra-subject variability in reaction times: Average and single-trial analyses. Biological Psychology, 87(1), 74-83. https://doi.org/10.1016/j.biopsycho.2011.02.005

Saville, C. W., Shikhare, S., Iyengar, S., Daley, D., Intriligator, J., Boehm, S. G., . . Klein, C. (2012). Is reaction time variability consistent across sensory modalities? Insights from latent variable analysis of single-trial P3b latencies. Biological Psychology, 91(2), 275-282. https://doi.org/10.1016/j.biopsycho.2012.07.006

Simmonds, D. J., Fotedar, S. G., Suskauer, S. J., Pekar, J. J., Denckla, M. B., \& Mostofsky, S. H. (2007). Functional brain correlates of response time variability in children. Neuropsychologia, 45(9), 2147-2157. https://doi.org/10.1016/j.neuropsychologia.2007.01.013

Tabachnick, B. G., \& Fidell, L. S. (2013). Using Multivariate Statistics (6 ed.). Boston: Pearson.

Tenke, C. E., \& Kayser, J. (2005). Reference-free quantification of EEG spectra: Combining current source density (CSD) and frequency principal components analysis (fPCA). Clinical Neurophysiology, 116(12), 2826-2846. https://doi.org/10.1016/j.clinph.2005.08.007

Tenke, C. E., Kayser, J., Abraham, K., Alvarenga, J. E., \& Bruder, G. E. (2015). Posterior EEG alpha at rest and during task performance: Comparison of current source density 
and field potential measures. International Journal of Psychophysiology, 97(3), 299309. https://doi.org/10.1016/j.ijpsycho.2015.05.011

Tenke, C. E., Kayser, J., Manna, C. G., Fekri, S., Kroppmann, C. J., Schaller, J. D., . . . Bruder, G. E. (2011). Current source density measures of electroencephalographic alpha predict antidepressant treatment response. Biological Psychiatry, 70(4), 388394. https://doi.org/10.1016/j.biopsych.2011.02.016

Tenke, C. E., Kayser, J., Pechtel, P., Webb, C. A., Dillon, D. G., Goer, F., . . Bruder, G. E. (2017). Demonstrating test-retest reliability of electrophysiological measures for healthy adults in a multisite study of biomarkers of antidepressant treatment response. Psychophysiology, 54(1), 34-50. https://doi.org/10.1111/psyp.12758

Tenke, C. E., Kayser, J., Svob, C., Miller, L., Alvarenga, J. E., Abraham, K., . . . Bruder, G. E. (2017). Association of posterior EEG alpha with prioritization of religion or spirituality: A replication and extension at 20-year follow-up. Biological Psychology, 124, 79-86. https://doi.org/10.1016/j.biopsycho.2017.01.005

Verleger, R., Jaśkowski, P., \& Wascher, E. (2005). Evidence for an integrative role of P3b in linking reaction to perception. Journal of Psychophysiology, 19(3), 165-181. https://doi.org/10.1027/0269-8803.19.3.165 


\section{Author Notes}

This research was conducted with the support of an Australian Government Research Training Program Scholarship to DK. GZS's contribution was supported by funding from a National Health and Medical Research Council (NHMRC)-Australian Research Council (ARC) Dementia Research Development Fellowship (GNT1102532). 


\section{Tables}

Table 1

Go ERP Component Correlates of Behavioural Outcomes (Pearson's r)

\begin{tabular}{lcccc}
\hline $\begin{array}{l}\text { Behavioural } \\
\text { Outcomes }\end{array}$ & $\begin{array}{c}\text { Central } \\
\mathrm{P} 2\end{array}$ & $\begin{array}{c}\text { Frontal } \\
\mathrm{N} 2 \mathrm{c}\end{array}$ & $\begin{array}{c}\text { Posterior-Left } \\
\text { P3b }\end{array}$ & $\begin{array}{c}\text { Central } \\
\text { SW }\end{array}$ \\
\hline Go Omissions & .13 & .19 & .28 & -.11 \\
Go mean RT & $.57^{*}$ & .27 & $-.45^{*}$ & -.26 \\
Go RTV & $.46^{*}$ & -.11 & -.06 & .01 \\
\hline
\end{tabular}

$*$ denotes significant one-tailed correlations with $p<.05$. Light grey shading indicates an expected finding that is consistent with the original study and dark grey shading marks a relationship we expected but did not find. No shading represents a new finding. 
USING PCA TO EXAMINE EEG RELATIONS TO PERFORMANCE 30

Table 2

EC EEG Predictors of Go/NoGo Task Responses

\begin{tabular}{lccc}
\hline & delta-1 & delta/theta & alpha-3 \\
& $\beta(t)$ & $\beta(t)$ & $\beta(t)$ \\
\hline Mean RT & $-.45(-2.14)$ & & \\
Go P3b & & $.42(1.96)$ & $.54(2.72)$ \\
\hline
\end{tabular}




\section{Figure Captions}

Figure 1. Grand mean Go ERPs at Fz, $\mathrm{Cz}$ and Pz, for the t-PCA input (full lines) and output data (dashed lines) are displayed in the top panel. t-PCA factor details, component topographies and loadings are shown in the bottom panels.

Figure 2. The top panel shows the grand mean NoGo ERPs at the midline sites, for the tPCA input (full lines) and output data (dashed lines). The panels below present the t-PCA factor details, component topographies and scaled loadings.

Figure 3. EEG spectral amplitudes, at the midline sites, for the eyes-closed and eyes-open states are shown in the top panel. The bottom panels display the f-PCA derived EEG component headmaps and the topographic and spectral similarities (as determined by Pearson's $r$ and the congruence $r_{\mathrm{c}}$ coefficients, respectively) between the factors obtained from the separate f-PCAs. Delta-1, delta/theta, alpha-1 and beta-2 components were identified as being fairly similar between the datasets. 


\section{Supplementary Materials}

\section{S1. Results}

\section{S1.1 EEG f-PCA Comparisons}

Figure S1 displays the f-PCA factor output from the initial dataset and compares this to the output obtained from the separate EC and EO f-PCAs. On the left, factor loadings are represented in microvolts, scaled by multiplying the loading at each frequency-point with the standard deviation of the EEG spectral amplitude at that point. The dashed lines through each f-PCA loading compare the peak frequencies across the datasets. Upon examination of the factor loadings, variance was misallocated between the conditions in the initial f-PCA. All three alpha components differ in peak frequency between the separate f-PCAs. While the EC component peaks appear to match those obtained from the initial f-PCA, these peaks have clearly shifted by $1 \mathrm{~Hz}$ with EO. This can also be seen with the beta-1 component; its $15 \mathrm{~Hz}$ peak can be seen in the initial and EC f-PCAs but this changes to $18 \mathrm{~Hz}$ with EO. The shape and amplitudes of the scaled factor loadings also appear to differ between EC and EO. When compared with the initial f-PCA loadings, data were underestimated for EC and overestimated for EO. The output obtained from the combined EC and EO f-PCA therefore imposes inaccurate frequencies for EO alpha and beta-1 components, and artificially increases the component amplitudes calculated for EO. As the only source of variance in the separate f-PCAs was from within the condition, this output was determined to better estimate the data and was used in subsequent analyses. 


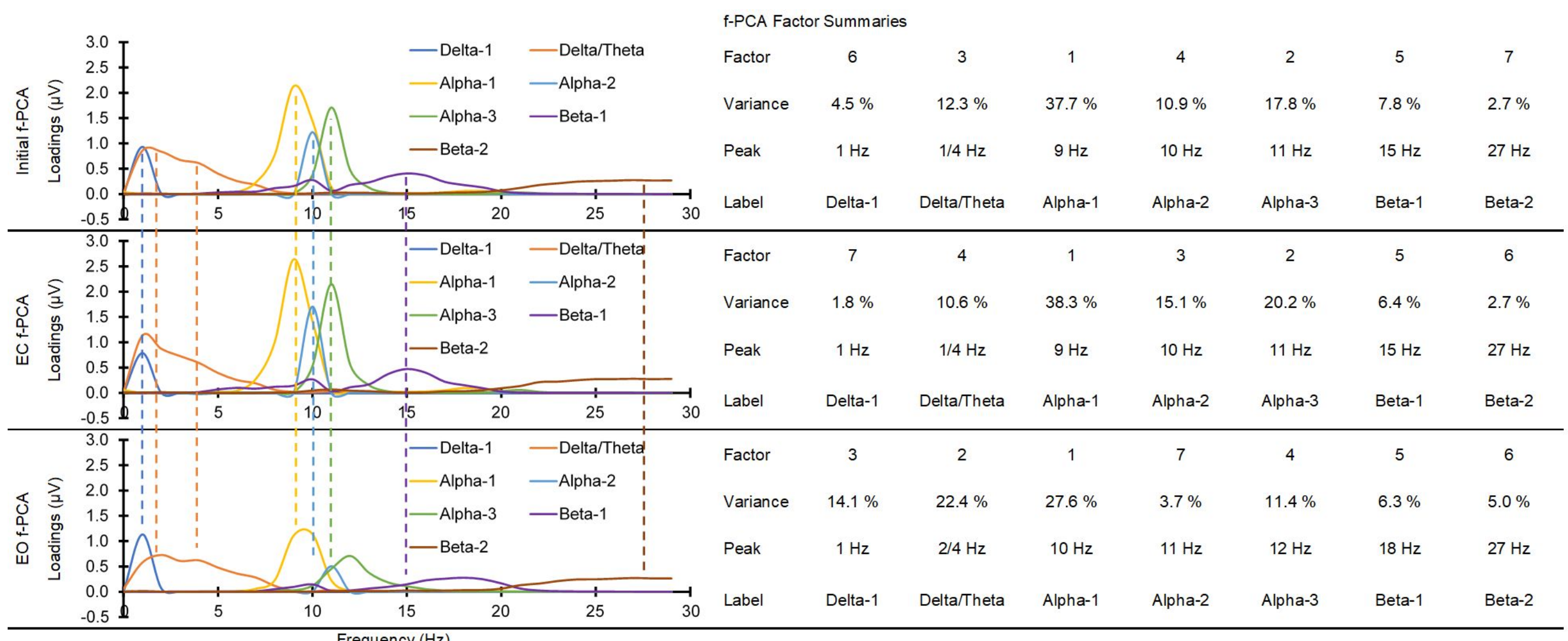

Figure S1. The output from the f-PCAs conducted with the combined EC and EO EEGs and the f-PCAs conducted separately on these resting state EEGs. Dashed lines visualise the misallocated variance between the EC/EO conditions in the initial f-PCA. Factor information from each f-PCA is displayed on the right. 


\section{S1.2 EEG Component Topographies}

Table S1 shows the topographic MANOVA outcomes for the EEG components for the EC state and the change from EC to EO. The top half of the table presents EC topography and the bottom half displays outcomes for EC to EO reactivity. With EC, delta-1 showed larger amplitudes fronto-parietally, especially in the frontal-right region, and was dominant across the midline; delta/theta amplitudes were also midline dominant, especially at the vertex. Thus, for delta- 1 and delta/theta, activity was pooled across $\mathrm{FCz}, \mathrm{Cz}, \mathrm{CPz}$. All three alphas, and beta-1, were posterior dominant, and so the region of interest was defined as the average over P3, Pz, P4. These components also showed less activity centrally, particularly on the left for alpha-2 and in the hemispheres for alpha-3, and greater midline amplitudes for alpha-1/2 and beta-1, with a parietal-right enhancement of beta-1 amplitude also evident. Beta- 2 was dominant in the midline, particularly centrally, and showed central activity in the left hemisphere; the midline region was pooled for beta-2.

EC to EO reactivity was defined by an overall increase in delta-1 amplitude; as no specific region was identified here, the midline region was selected $(\mathrm{FCz}, \mathrm{Cz}, \mathrm{CPz})$.

Delta/theta amplitude decreased parietally, largely in the midline and at the vertex, and so the midline region was also selected for this component. Alpha-1 showed a strong parietal decrease that contributed to a larger fronto-parietal $c f$. central mean; the parietal region (P3, $\mathrm{Pz}, \mathrm{P} 4)$ was selected for analysis. Beta-2 amplitude increased from EC to EO in the hemispheres, especially in the frontal hemispheres (F3, F4).

Table S1

Topographic MANOVA Outcomes for Resting State Activity

\begin{tabular}{llrrr}
\hline Eyes Closed & & \multicolumn{1}{c}{$F$} & \multicolumn{1}{c}{$p$} & $\eta_{p}{ }^{2}$ \\
\hline Band & Effect & 8.40 & .009 & .31 \\
\multirow{3}{*}{ Delta-1 } & $\mathrm{C}<\mathrm{F} / \mathrm{P}$ & 18.81 & $<.001$ & .50 \\
& $\mathrm{M}>\mathrm{L} / \mathrm{R}$ & 5.41 & .031 & .22 \\
\hline \multirow{2}{*}{ Delta/Theta } & $\mathrm{F}>\mathrm{P} \times \mathrm{L}<\mathrm{R}$ & 73.57 & $<.001$ & .79 \\
& $\mathrm{M}>\mathrm{F} / \mathrm{P}$ & 6.30 & .021 & .25 \\
\hline
\end{tabular}




\begin{tabular}{llrrr}
\hline \multirow{3}{*}{ Alpha-1 } & $\mathrm{F}<\mathrm{P}$ & 10.64 & .004 & .36 \\
& $\mathrm{C}<\mathrm{F} / \mathrm{P}$ & 6.20 & .022 & .25 \\
& $\mathrm{M}>\mathrm{L} / \mathrm{R}$ & 9.45 & .006 & .33 \\
\hline \multirow{4}{*}{ Alpha-2 } & $\mathrm{F}<\mathrm{P}$ & 13.85 & .001 & .42 \\
& $\mathrm{C}<\mathrm{F} / \mathrm{P}$ & 11.31 & .003 & .37 \\
& $\mathrm{M}>\mathrm{L} / \mathrm{R}$ & 11.53 & .003 & .38 \\
& $\mathrm{C}<\mathrm{F} / \mathrm{P} \times \mathrm{L}>\mathrm{R}$ & 4.49 & .047 & .19 \\
\hline \multirow{3}{*}{ Alpha-3 } & $\mathrm{F}<\mathrm{P}$ & 32.32 & $<.001$ & .63 \\
& $\mathrm{C}<\mathrm{F} / \mathrm{P}$ & 8.84 & .008 & .32 \\
& $\mathrm{C}<\mathrm{F} / \mathrm{P} \times \mathrm{M}>\mathrm{L} / \mathrm{R}$ & 9.09 & .007 & .32 \\
\hline \multirow{5}{*}{ Beta-1 } & $\mathrm{F}<\mathrm{P}$ & 16.31 & .001 & .46 \\
& $\mathrm{C}<\mathrm{F} / \mathrm{P}$ & 15.69 & .001 & .45 \\
& $\mathrm{~L}>\mathrm{R}$ & 9.08 & .007 & .32 \\
& $\mathrm{M}>\mathrm{L} / \mathrm{R}$ & 9.26 & .007 & .33 \\
Beta-2 & $\mathrm{F}<\mathrm{P} \times \mathrm{L}>\mathrm{R}$ & 5.01 & .037 & .21 \\
\hline $\mathrm{M}>\mathrm{L} / \mathrm{R}$ & 12.36 & .002 & .39 \\
& $\mathrm{C}<\mathrm{F} / \mathrm{P} \times \mathrm{L}<\mathrm{R}$ & 8.74 & .008 & .32 \\
& $\mathrm{C}>\mathrm{F} / \mathrm{P} \times \mathrm{M}>\mathrm{L} / \mathrm{R}$ & 19.01 & $<.001$ & .50 \\
\hline
\end{tabular}

EC to EO Reactivity

\begin{tabular}{llrrr}
\hline Delta-1 & EC $<$ EO & 86.73 & $<.001$ & .82 \\
\hline \multirow{5}{*}{ Delta/Theta } & EC $>$ EO & 340.52 & $<.001$ & .95 \\
& EC $>$ EO $\times \mathrm{F}<\mathrm{P}$ & 5.31 & .033 & .22 \\
& EC $>$ EO $\times \mathrm{M}>\mathrm{L} / \mathrm{R}$ & 15.87 & .001 & .46 \\
& $\mathrm{EC}>\mathrm{EO} \times \mathrm{C}>\mathrm{F} / \mathrm{P} \times \mathrm{M}>\mathrm{L} / \mathrm{R}$ & 8.58 & .009 & .31 \\
\hline \multirow{3}{*}{ Alpha-1 } & $\mathrm{EC}>\mathrm{EO}$ & 30.27 & $<.001$ & .61 \\
& $\mathrm{EC}>\mathrm{EO} \times \mathrm{F}<\mathrm{P}$ & 7.33 & .014 & .28 \\
& $\mathrm{EC}>\mathrm{EO} \times \mathrm{C}<\mathrm{F} / \mathrm{P}$ & 6.16 & .023 & .24 \\
\hline \multirow{3}{*}{ Beta-2 } & $\mathrm{EC}<\mathrm{EO} \quad 2.76$ & .113 & .13 \\
& $\mathrm{EC}<\mathrm{EO} \times \mathrm{M}<\mathrm{L} / \mathrm{R}$ & 5.32 & .033 & .22 \\
& $\mathrm{EC}<\mathrm{EO} \times \mathrm{F}>\mathrm{P} \times \mathrm{M}<\mathrm{L} / \mathrm{R}$ & 13.78 & .002 & .41 \\
\hline
\end{tabular}


Grandy Nhe arfictogy ERPs
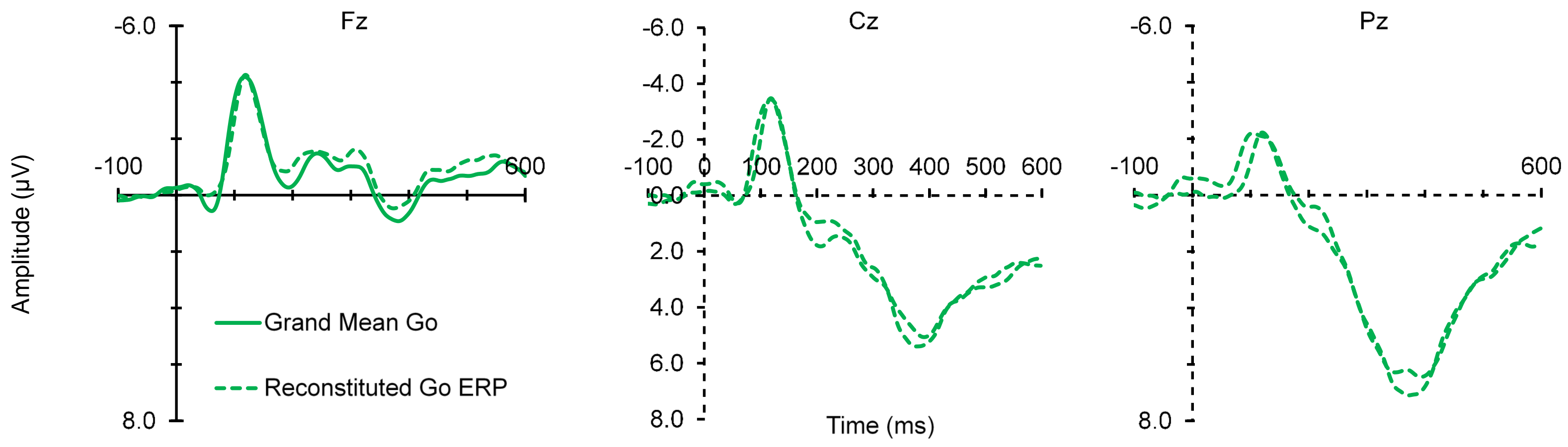

Factor

Variance (\%)

5

3

Latency (ms)

3.8

8.0

6

2

4

1

Label

118

168

2.1

26.0

6.4

42.8

224

312

394

564

P2

N2c
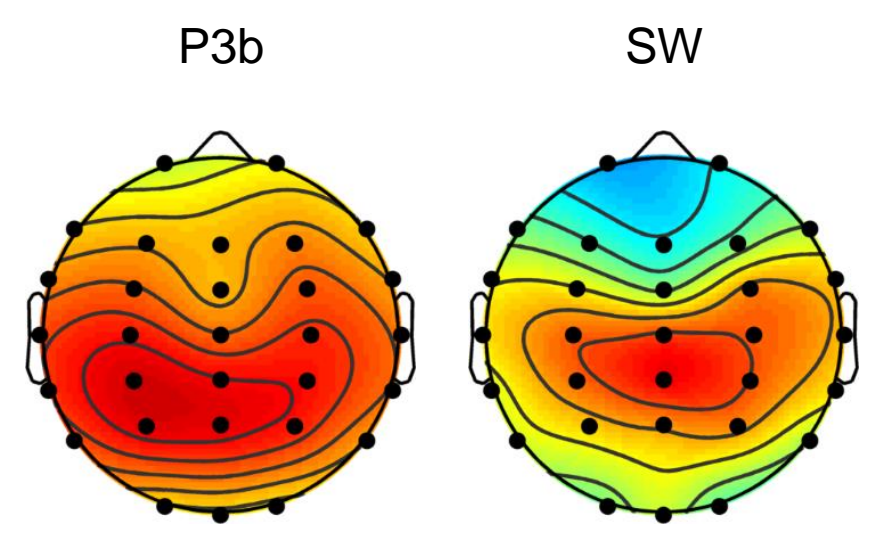

$\mu \mathrm{V}$

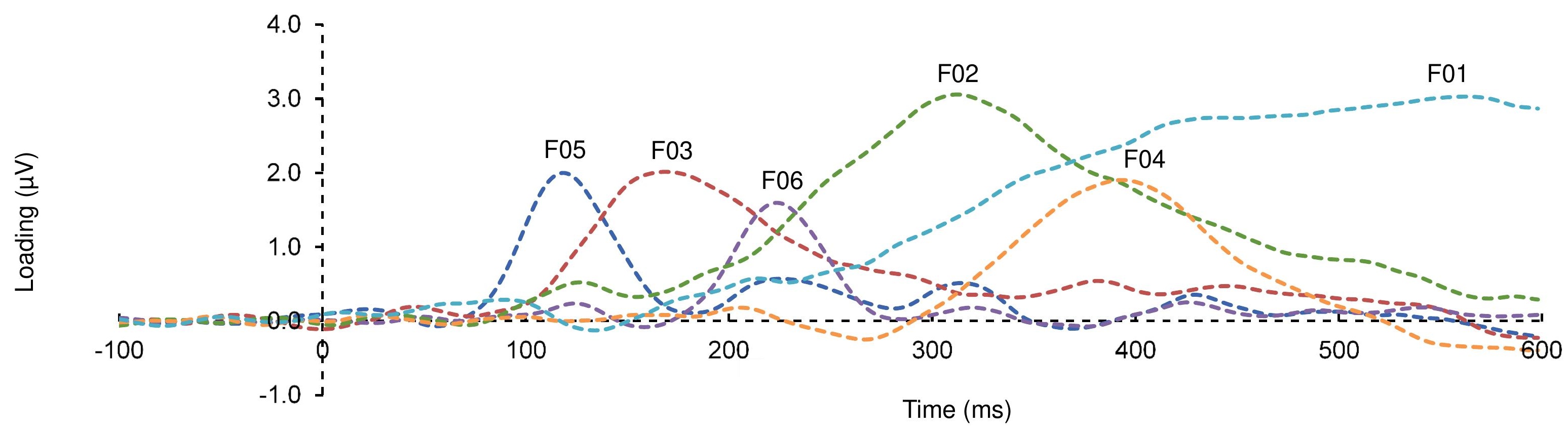


Grand Rieapp Norgor ERPs
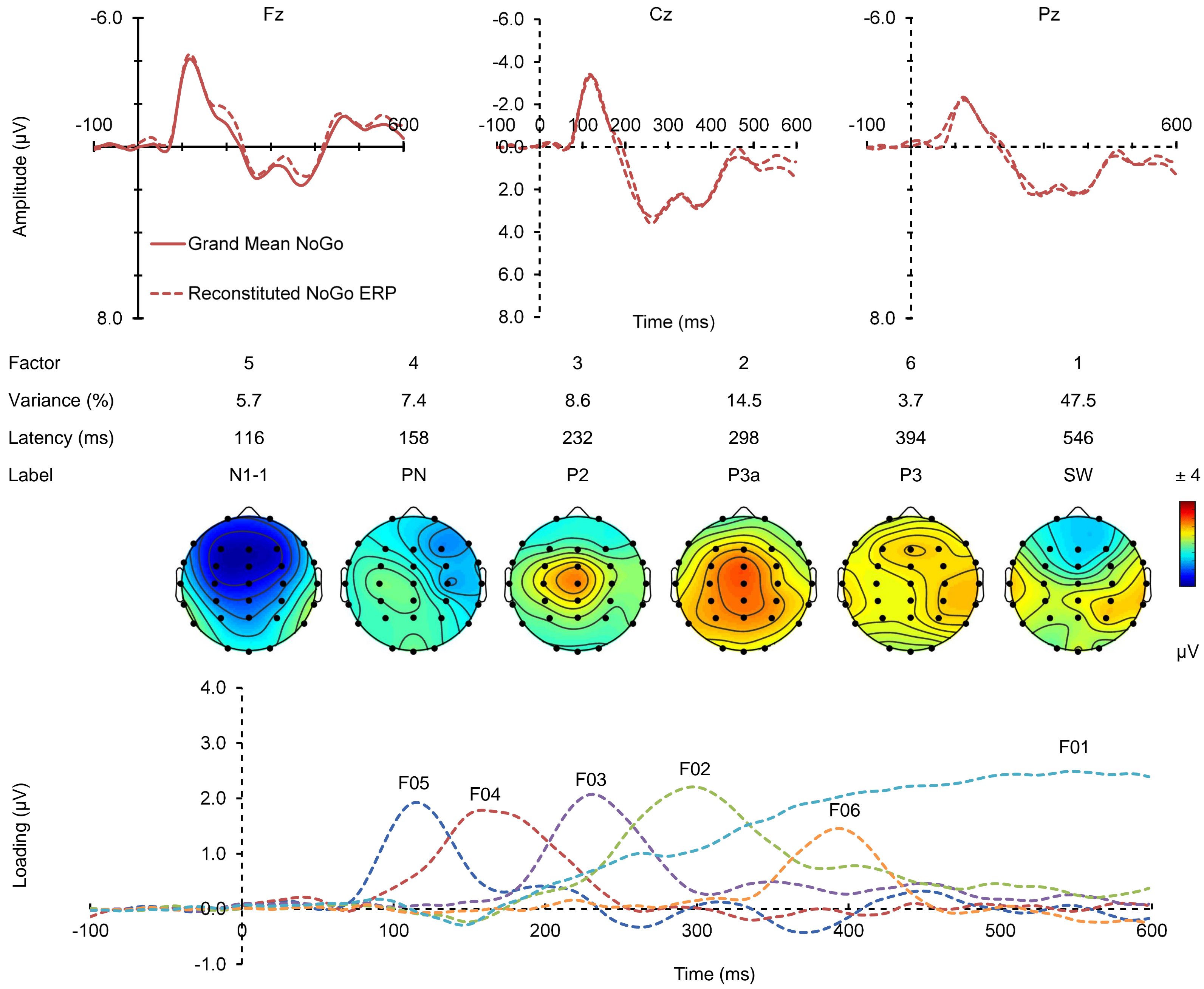

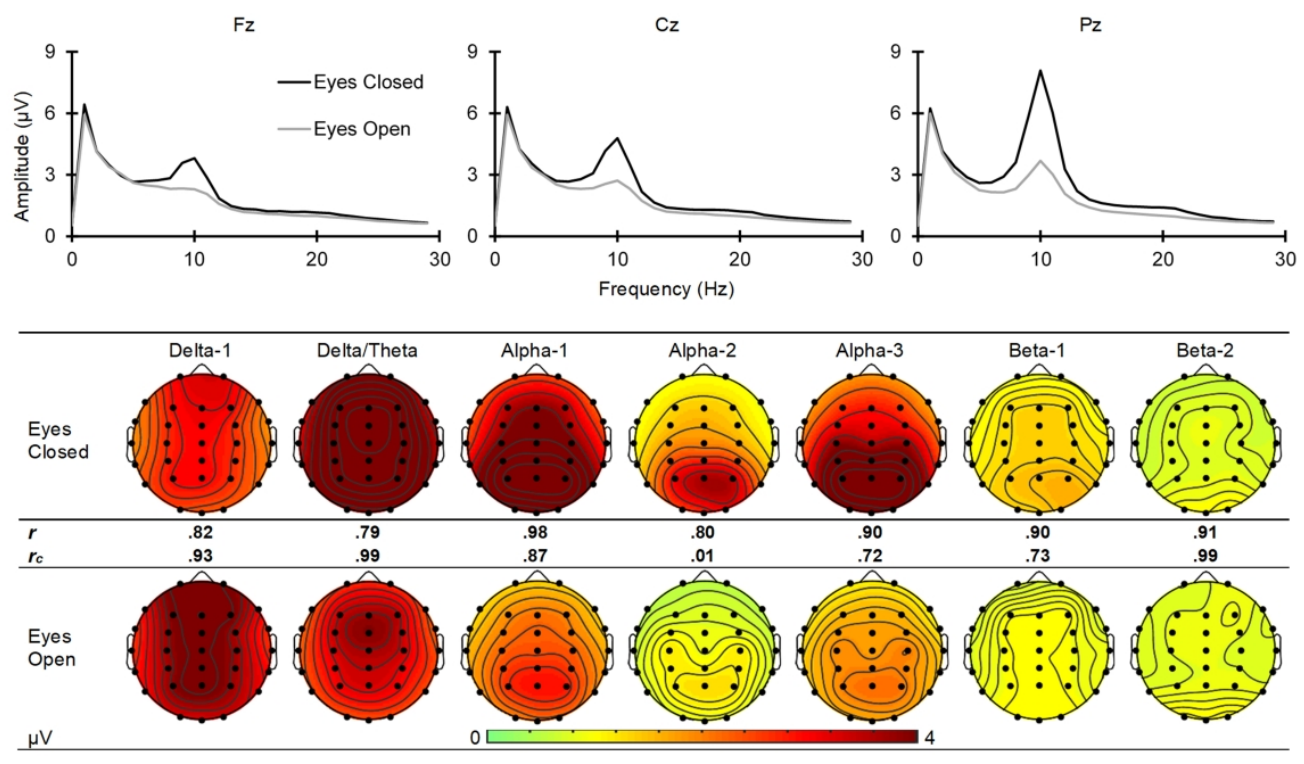

$279 \times 159 \mathrm{~mm}(600 \times 600 \mathrm{DPI})$ 